\title{
Sistemas de información geográfica y modelado hidráulico de redes de abastecimiento de agua potable: estudios de caso en la provincia de Guanacaste, Costa Rica
}

\author{
Geographic information systems and hydraulic modeling of \\ potable water supply networks: case studies in the province of \\ Guanacaste, Costa Rica
}

\author{
Esteban Alberto González-Ramírez ${ }^{1}$ \\ Esteban Bejarano-Salazar ${ }^{2}$ \\ Instituto Costarricense de Acueductos y Alcantarillados, Costa Rica
}

\begin{abstract}
Resumen
Las metodologías de diseño y análisis de sistemas de acueductos y alcantarillados han ido cambiando con el surgir de nuevas herramientas y tecnologías que, además de simplificar estos procesos, resultan cada vez más confiables y precisas. La aplicación directa de estas nuevas tecnologías ha mejorado notablemente la manera en que se planifican, se diseñan y construyen los proyectos de agua potable y saneamiento en el país. El modelado hidráulico de redes es una metodología que se viene aplicando desde hace varios años, tanto para el análisis y diseño de redes de agua potable como para redes de alcantarillado. Las versiones más recientes de software de modelado hidráulico tienen la enorme ventaja de que son compatibles con Sistemas de Información Geográfica (SIG). En la presente investigación se muestra un procedimiento teórico y metodológico utilizado para simplificar el modelado hidráulico a través de la captura y sistematización de los datos en un SIG, además de una serie de resultados obtenidos de casos específicos en sistemas de la provincia de Guanacaste en Costa Rica.
\end{abstract}

1 Licenciado en Ciencias Geográficas, pertenece a la Subgerencia de Ambiente, Investigación y Desarro1lo del Instituto Costarricense de Acueductos y Alcantarillados, San José, Costa Rica. Correo electrónico: egonzalez@aya.go.cr

2 Licenciado en Ingeniería Civil, pertenece a la Subgerencia de Ambiente, Investigación y Desarrollo del Instituto Costarricense de Acueductos y Alcantarillados, San José, Costa Rica. Correo electrónico: ebejarano@, aya.go.cr 
Palabras clave: Sistemas de Información Geográfica (SIG), Modelado Hidráulico, redes de distribución de agua, acueductos.

\begin{abstract}
Design and analysis methodologies for aqueduct and sewerage systems have been changing with the emergence of new tools and technologies that, in addition to simplifying these processes, are increasingly reliable and precise. The direct application of these new technologies has greatly improved the way in which drinking water and sanitation projects are planned, designed and built in the country. Hydraulic modeling of networks is a methodology that has been applied for several years, both for the analysis and design of drinking water networks and sewage networks. The most recent versions of hydraulic modeling software have the enormous advantage of being compatible with Geographic Information Systems (GIS). The present investigation shows a theoretical and methodological procedure used to simplify hydraulic modeling through the capture and systematization of data in a GIS, as well as a series of results obtained from specific cases in network systems throughout the province of Guanacaste in Costa Rica.
\end{abstract}

Keywords: Geographic Information Systems (GIS); hydraulic modeling; water distribution networks; aqueducts.

\title{
Introducción
}

Los sistemas de abastecimiento de agua potable, así como los que dan tratamiento a aguas residuales han sido difíciles de manejar y gestionar en todo su desarrollo histórico. Uno de los principales problemas en la actualidad es la gran cantidad de información desagregada sobre la infraestructura instalada, lo que genera errores y atrasos en la planeación y gestión de nuevas obras. Una moderna gestión de este tipo de sistemas requiere cada vez más del uso de recursos tecnológicos y manejo adecuado de los datos, en donde los Sistemas de Información Geográfica (SIG) adquieren un papel preponderante en la captura, almacenamiento, manipulación, análisis y presentación de la información espacial existente.

En esta misma línea tecnológica, se hace cada día más común el uso de software especializado para modelado hidráulico de redes de agua potable o aguas residuales. Los modelos hidráulicos se utilizan hoy en día con mucha frecuencia para el análisis y diseño de sistemas de distribución de agua, ya que permiten ingresar diferentes tipos de datos y combinarlos para obtener una simulación bastante precisa del comportamiento del sistema real que se está analizando.

Con este auge tecnológico y el desarrollo de modernos programas informáticos aplicados a la gestión de datos sobre redes de agua se puede tener acceso rápido y confiable a información especializada. Los modelos 
hidráulicos correctamente construidos y calibrados pueden ayudar notablemente a optimizar los sistemas, mejorar las condiciones de planeación de nuevas infraestructuras, así como servir de base para labores operativas y de mantenimiento.

Los esfuerzos institucionales realizados para tener datos actualizados de sus sistemas demuestran que siempre ha existido la necesidad de contar con información fiable con respecto a la infraestructura instalada. Las planotecas y centros técnicos especializados institucionales cuentan con un vasto almacenamiento de datos, principalmente análogos (en papel, pergaminos, entre otros), los cuales fueron constituidos en las décadas de los70, 80, inclusive 90, cuando la cartografía en su gran mayoría se realizaba sobre papel, utilizando como referencia mapas a escala "mediana" como lo son las hojas topográficas 1:50000 del Instituto Geográfico Nacional de Costa Rica (IGN), o en algunos casos especiales levantamientos topográficos convencionales.

Con el avance en programas computacionales se incorpora la cartografía digital para la representación de la mayor parte de la infraestructura de acueductos y alcantarillados, principalmente en sistemas CAD (Computer-Aided Design) o diseño asistido por computadora, lo cual ha permitido un manejo más ágil de los datos, pero que acarrea una serie de problemáticas, ya que inicialmente estos programas no fueron construidos para cartografía, sino más bien para diseño gráfico ingenieril y de arquitectura, en un plano de dos dimensiones, lo que ha generado que dicha cartografía tenga distorsiones de proyección cartográfica. Actualmente, los CAD han avanzado a programas que incluyen aspectos SIG, por ejemplo, Autodesk Map 3D.

En este documento se muestran los aspectos desarrollados en el proceso, primeramente, una sección con aspectos teóricos fundamentales y los objetivos del proyecto. En una segunda parte se muestra los métodos, técnicas y pasos para la obtención de los productos. En un tercer apartado se muestran algunos de los productos finales logrados con la aplicación de la metodología, además de una serie de conclusiones. Finalmente, las referencias bibliográficas. 


\section{Área de estudio}

Mapa 1. Ubicación de sistemas en estudio.

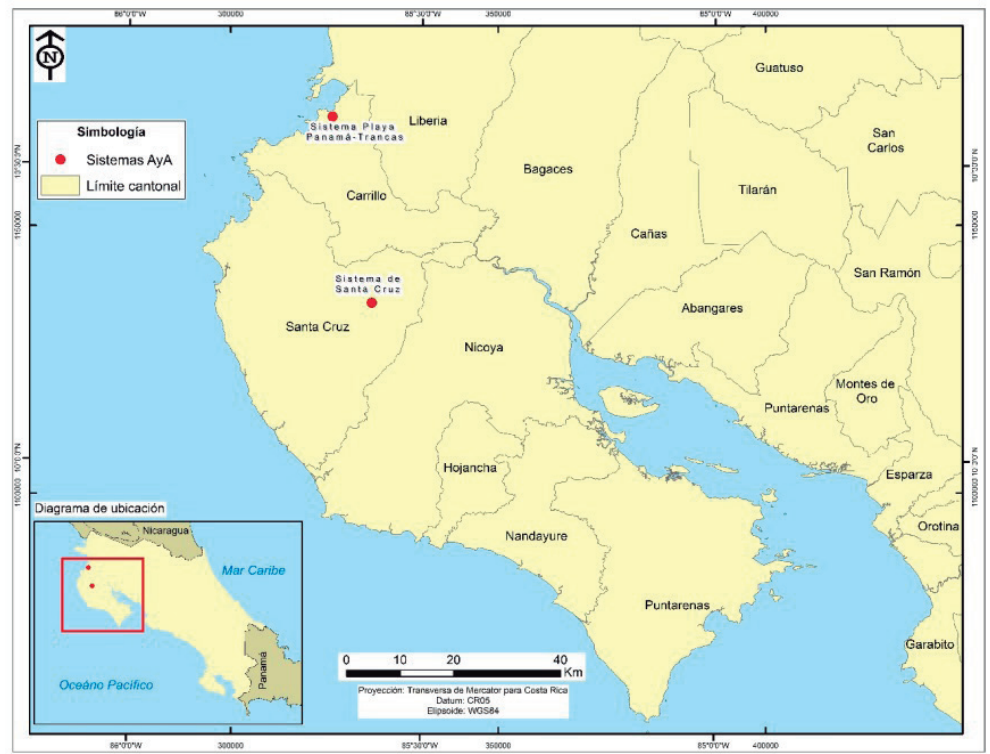

Fuente: ICAA 2017 (Departamento de Desarrollo Físico, UEN Programación y Control) e ITCR 2014 (Atlas cantonal digital).

La aplicación de la metodología de modelado hidráulico basada en datos creados y preparados con el uso de tecnologías de Sistemas de Información Geográfica (SIG) se llevó a cabo para el sistema de abastecimiento de agua potable de la ciudad de Santa Cruz, en el cantón del mismo nombre, y se planea utilizarla posteriormente para el nuevo sistema de abastecimiento del sector Papagayo Norte (específicamente sector Playa Panamá - Trancas) ubicado en el cantón de Carrillo, ambos en la provincia de Guanacaste. Ver mapa 1.

\section{Objetivos}

Objetivo general

- Desarrollar e implementar una metodología para el modelado hidráulico de redes de distribución de agua, basada en el uso de información creada mediante el uso de Sistemas de Información Geográfica. 


\section{Objetivos especificos}

- Implementar una serie de técnicas SIG y de modelado hidráulico de redes para la planeación y optimización de sistemas de agua potable y saneamiento.

- Incorporar la información técnica (gráfica y temática) correspondiente a la red de agua potable de los sistemas en estudio en un SIG y realizar las respectivas transformaciones geométricas.

- $\quad$ Integrar en una base de datos SIG la información técnica de los sistemas en estudio.

- Construir y calibrar un modelo hidráulico para simular el comportamiento de redes de distribución de agua.

\section{Marco Teórico}

Este proyecto se categoriza como cuantitativo, ya que se basó en aspectos observables y capaces de medirse. Para la obtención de los mapas, bases de datos, modelos, y los demás resultados se utilizaron datos numéricos y gráficos, específicos de cada sistema, o área territorial de influencia, en un Sistema de Información Geográfica y a un programa de simulación hidráulica en el que, mediante técnicas especializadas, se gestionaron y visualizaron los datos en forma gráfico-espacial. En dicho contexto, en el caso de la planificación de los recursos hídricos en las obras para acueductos y sistemas de saneamiento, la información resulta imprescindible, ya que permite capturar, almacenar, manipular, analizar, modelar y presentar datos espacialmente referenciados (Foresman, 1998), cuyo papel es indiscutible en la implementación para obras de este tipo (acueductos y sistemas de saneamiento), es decir, obras con fuerte impacto socio natural.

Un modelo es una representación de la realidad, el cual se genera mediante la selección y simplificación de sus partes. Para que sea geográfico, el modelo debe tener un sistema de referenciación (Rodríguez, 2008). El modelo geográfico de la realidad en un SIG se caracteriza por separar las cosas y los seres que hay en el territorio, descomponiéndolos en partes. Estas partes son el resultado de una disección lógica y consistente de la realidad, que al segregarla se obtienen solamente algunos hechos o aspectos. En conjunto, significa concebir un modelo de la realidad y diseñar una base de datos para contenerlo (Moreno, 2008). Este aspecto se evalúa a partir de un análisis de capacidad hidráulica en sistemas AyA, y 
distribución espacial diferenciada de fenómenos y procesos en el territorio. Cuando se representan los fenómenos geográficos mediante puntos, líneas y polígonos, como en el caso de esta investigación, se utiliza el denominado modelo de datos vectorial. El modelo vectorial ha sido la base de la cartografía analógica clásica, pues resulta particularmente útil para representar entidades geográficas discretas (Rodríguez, 2008).

En el caso de la información alfanumérica (tablas que almacenan los datos), esta permite hablar de entidades geográficas y no de meros objetos gráficos, mostrando de aquéllas sus valores cualitativos y cuantitativos, y ofreciendo así la posibilidad de establecer jerarquías y operaciones matemáticas. Además, los procesos realizados con las tablas de una base de datos están limitados solo por la cantidad de información disponible (Cervera y Rodríguez, 2008).

Las herramientas de análisis espacial que proporcionan los SIG, como la superposición de polígonos, los análisis de proximidad, la generación de modelos o las simulaciones, tienen un papel clave, ya que le brindan a los/as especialistas la oportunidad de mejorar los resultados en sus estudios y proyectos (Comas, 1993). Con la información bien organizada en la base de datos espaciales, el SIG nos faculta para desarrollar un rico abanico de tratamiento y análisis (O`Sullivan y Unwin, 2003).

\section{Metodología}

Proceso técnico-metodológico en el Sistema de Información Geográfico (SIG).

En las últimas décadas, se han desarrollado desde la cosmovisión de la geografía cuantitativa una serie de aplicaciones y herramientas informáticas, denominadas Sistemas de Información Geográfica (SIG), las cuales se han convertido en una tecnología básica y de gran poder, para capturar, almacenar, manipular, analizar, modelar y presentar datos espacialmente referenciados.

La moderna cartografía con la que cuenta el país con base al PRCR (Programa de Regularización del Catastro y el Registro) y accesible a las instituciones públicas, ha venido a mejorar y llenar un espacio en la homogenización de la información generada. Es a través de esta nueva información que se ha venido trabajando para la creación de nuevos mapas de acueductos y alcantarillados. 


\section{Incorporación de la información gráfica.}

Como se mencionó en apartados anteriores, existe una amplia base de información técnica y gráfica sobre los sistemas de abastecimiento de agua potable y saneamiento en el AyA, como lo son los levantamientos sobre mapas del IGN, sobre censales del INEC (Instituto Nacional de Estadística y Censo), y otros, los cuales en su mayoría han sido trasladados digitalmente, principalmente en versiones CAD.

El primer aspecto desarrollado en este proyecto es la búsqueda de la información perteneciente al área de estudio, ya sea en versión digital u otra. En este caso para los sistemas en estudio existe información disponible en versión digital. Es a partir de esta información digital (en CAD) que se inicia el proceso cartográfico que permite la creación de datos más confiables y que se utilizan para el modelado hidráulico (ver Figura 1). El trabajo se realiza en el software de la empresa ESRI ArcGIS 10.

La información del CAD se traslada al SIG, en el cual se le deben de realizar una serie de transformaciones geométricas para realizar la homologación con la cartografía del PRCR y ajustar a la proyección cartográfica oficial, la CRTM05. La transformación incluye primeramente convertir el CAD en el SIG a una entidad vectorial, es decir, un archivo tipo shapefile (SHP) que permita su edición en el programa, ya que originalmente se comporta como una imagen.

Figura 1. Ejemplo de la versión original de la información contenida en un CAD, en este caso del sistema de Santa Cruz de Guanacaste.

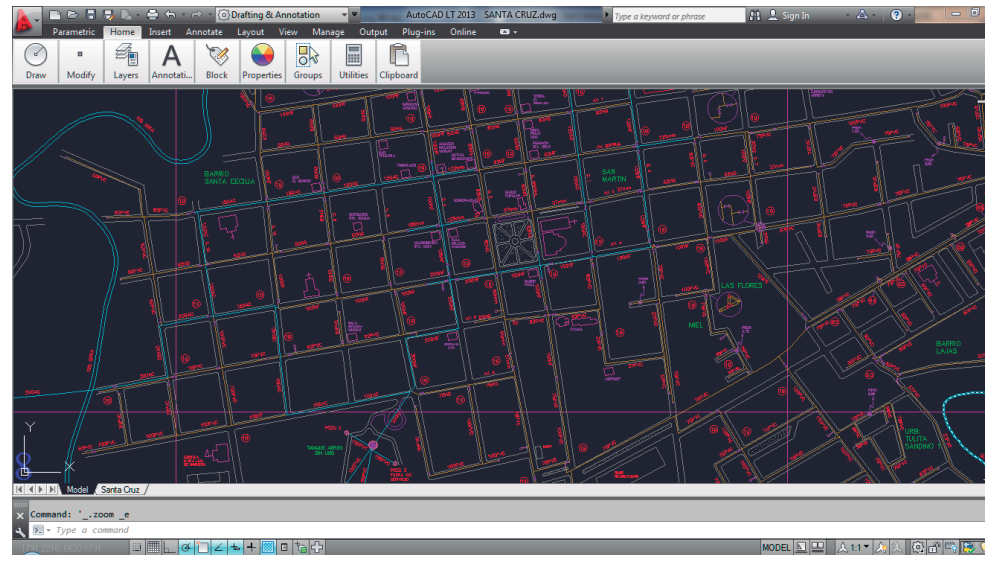

Fuente: AyA 2012. Departamento de Desarrollo Físico, UEN P y C. 
Como se observa en la Figura 2, a través de herramientas que posee el SIG, se realiza la transformación de un archivo CAD a SHP, dando la posibilidad de editarlo. El proceso continúa con la georeferenciación de los datos, que se realiza mediante la extensión Georeferencing, y también haciendo uso herramientas Editor y Data Management Tools-Projections and Transformation que posee el programa ArcGIS.

Figura 2. Proceso de transformación a shapefile.

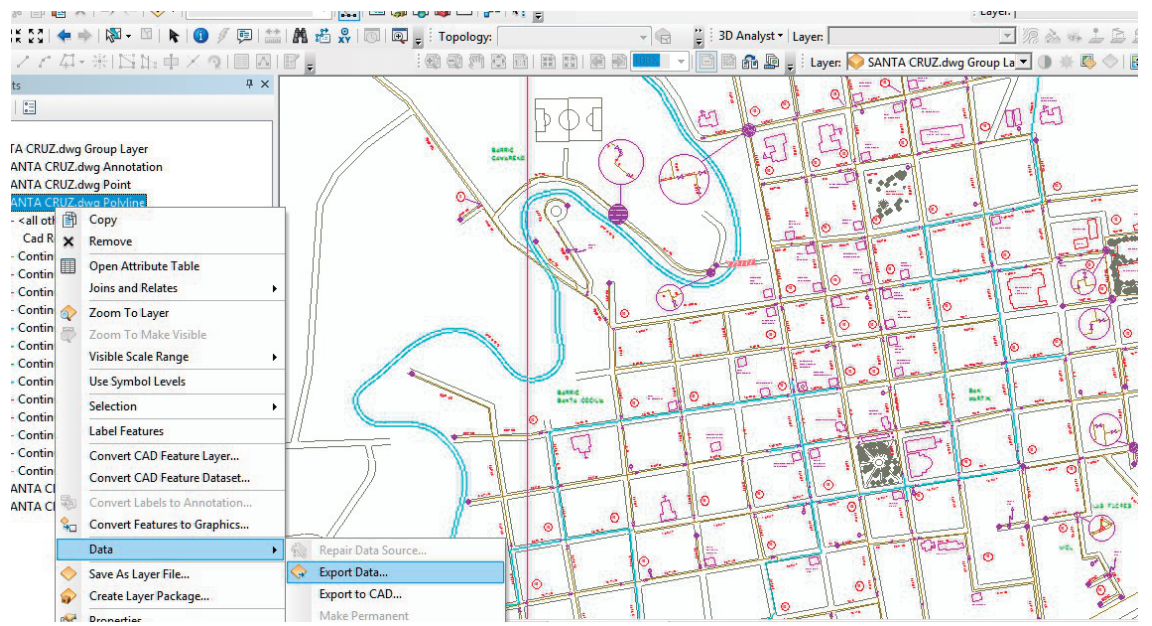

Fuente: AyA 2012. Departamento de Desarrollo Físico, UEN Programación y Control.

Al realizar la georreferenciación y proyección de los datos, nos encontramos con un desfase geométrico de la información con respecto a la cartografía oficial actual. Este desfase corresponde en parte a la inexistencia de cartografía adecuada en el momento de realizar los levantamientos de campo de los sistemas, además de que el uso de tecnologías como GPS (Sistema de Posicionamiento Global) y SIG no estaban disponibles. Otro aspecto que influye en el error geométrico es el uso de programas de diseño arquitectónico para creación de cartografía, los cuales no están construidos para proyectar la curvatura de la tierra como sí lo hace actualmente los SIG. El detalle descrito se observa en la Figura 4, donde se observa con azul la cartografía del PRCR (oficial mediante el anuncio por parte del Instituto Geográfico Nacional y el Registro Inmobiliario, Aviso No 012011: Oficialización de la cartografía básica escalas 1:1.000 y 1:5.000, 
publicado en el diario oficial La Gaceta $\mathrm{N}^{\circ} 146$ del viernes 29 de julio de 2011) y en color café la información del AyA. A partir de este momento se viene un trabajo de corrección manual mediante el Editor del ArcGIS de cada entidad geográfica.

Este trabajo es uno de los más laboriosos, ya que incluye toda una revisión de las tuberías e infraestructura representada. En general, este trabajo comprende las siguientes actividades:

- Creación y clasificación de capas de información por atributos (tuberías, tanques, válvulas, reducciones, hidrantes, otros).

- $\quad$ Reubicación en su posición real: esto significa mediante la interpretación de los mapas AyA y la nueva cartografía (incluyendo ortofotos), y la búsqueda de puntos de semejanza en el plano urbano y de vías, mover cada elemento a su posición considerada como verdadera.

- Limpieza de capas: incluye una revisión exhaustiva de las capas generadas, eliminando todas aquellas entidades que presentan duplicidad, que están dividas a pesar de pertenecer a un mismo elemento, que no están extendidas hasta puntos finales como la esquina de una manzana, entre otros detalles varios.

- $\quad$ Arreglo y construcción de la base de datos: cada capa o layer posee una base de datos, la cual debe ser revisada y corregida, eliminando datos que por default trae originalmente del CAD, además de agregar nuevas columnas que incluyan datos de diámetro, material, región, sistema, oficina que opera, etc.

- $\quad$ Reubicación de algunas entidades con respecto a puntos de control GPS tomados en el campo.

Otra de las actividades realizadas es la verificación de campo de algunos puntos de interés y su respectivo levantamiento mediante GPS, lo cual permite una mejor precisión en la localización de las principales infraestructuras.

En la Figura 3 se detalla un ejemplo de la tubería e infraestructura ya adaptadas geométricamente a las capas del PRCR. Este proceso también incluyó la verificación puntual de ubicación de cada válvula, reducción, tanque, pozo e hidrante del sistema. Cada entidad posee ahora la característica específica y una ubicación cercana a la realidad. 
Otro de los aspectos realizados en el área de estudio y que forman parte del insumo, tanto para el modelado hidráulico como para otros trabajos, como, por ejemplo, la localización óptima de estructuras o conflicto de uso de suelo es la creación de modelos digitales de elevación (MDE). La información generada por el PRCR contiene, además de la cartografía general y las ortofotos, una serie de archivos ASCII con valores puntuales DTM, el cual es un archivo que contiene datos para crear modelos digitales de terreno. Estos archivos contienen toda la información triangulada que define la superficie.

Figura 3. Detalle de capas de información del sistema Santa Cruz corregidas con respecto a cartografía oficial y puntos GPS.

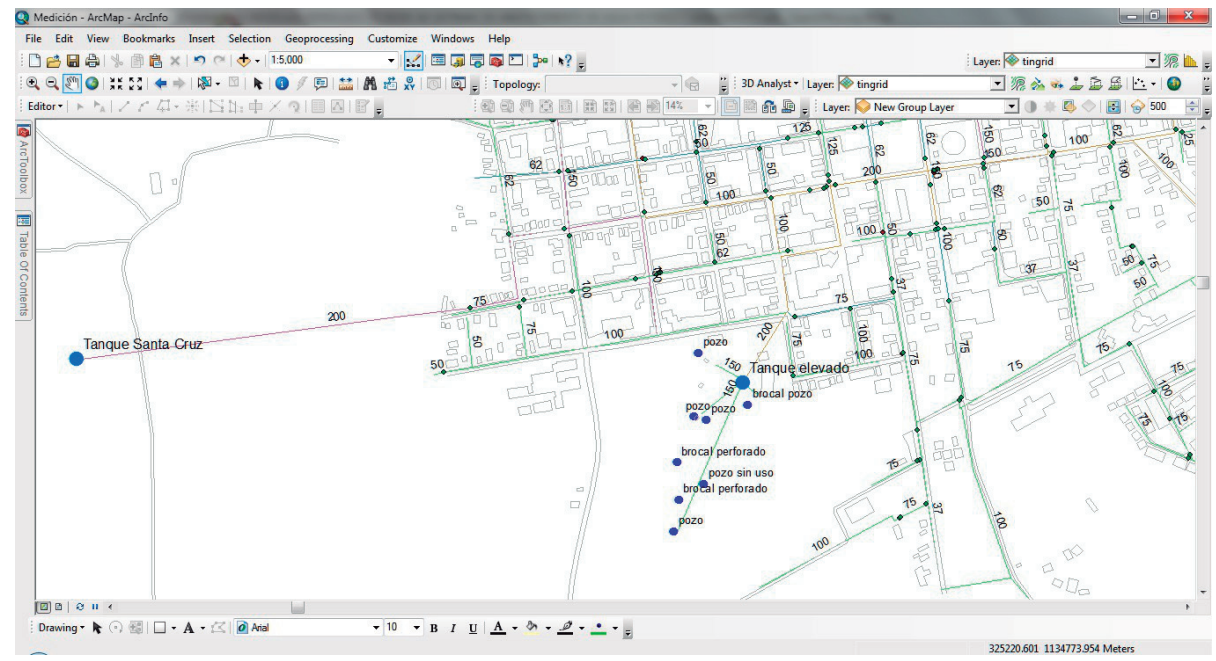

Fuente: AyA 2012. Departamento de Desarrollo Físico, UEN Programación y Control.

Un archivo DTM contiene datos de un modelo digital del terreno y una red de triángulos (creada al triangular los datos). Los archivos DTM son de doble precisión. No necesitan el archivo de dibujo en el que fueron creados para ser un archivo correcto. Con base a esta información, se construyeron mediante la extensión 3D Analyst y Spatial Analyst Tools del ArcGIS los MDE y curvas de nivel en donde se ubican los sistemas en estudio del AyA. 
A partir del Modelo Digital del Terreno, se generan curvas de nivel cada metro a partir de la herramienta Contour del 3D Analyst, lo cual es la representación más precisa del terreno de áreas relativamente grandes, tales como donde se ubican los sistemas. Para su visualización también se realiza una representación en el módulo ArcScene del ArcGIS, como se detalla en la Figura 4. Otro aspecto desarrollado fue la creación por manzana de polígonos con información de los servicios instalados y del consumo, los cuales dan la posibilidad de analizar la distribución espacial de diversos fenómenos y procesos, como el cálculo de densidades, estimación del consumo promedio, distribución espacial de servicios por categorías, entre otros.

Figura 4. Detalle de la visualización del Modelo Digital del Terreno en el área del sistema Santa Cruz en ArcScene.

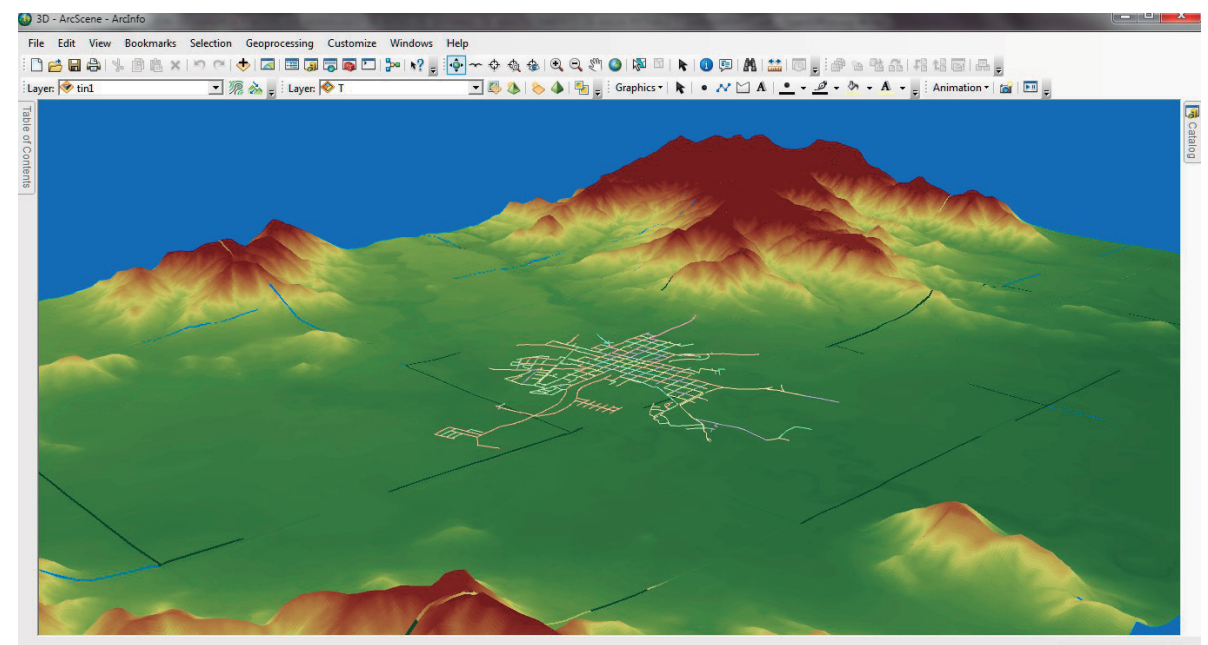

Fuente: AyA 2012. Departamento de Desarrollo Físico, UEN Programación y Control.

Como ejemplo de una aplicación de la información anterior se observa el Mapa 2, en donde se espacializan los datos de consumo total y promedio mensual del sistema Santa Cruz. El estudio de las distribuciones espaciales de un determinado fenómeno permite, en el caso de la densidad, ver disparidades en cuanto a la presión que tendrá la dotación de servicios, o puede dar idea de las áreas con deficiencias en el servicio, o definir elementos puntuales que sobresalen o que pueden tener algún aspecto que las 
diferencie, de manera que se puedan ubicar problemas en el sistema (Buzai: 2011). También a través de esto se pueden hacer estudios de asociaciones espaciales, como comparaciones mediante visualización o determinar de áreas homogéneas, y con ello se pueden zonificar áreas en específico, definidas por criterios especiales.

Mapa 2. Distribución del consumo total promedio en Santa Cruz.

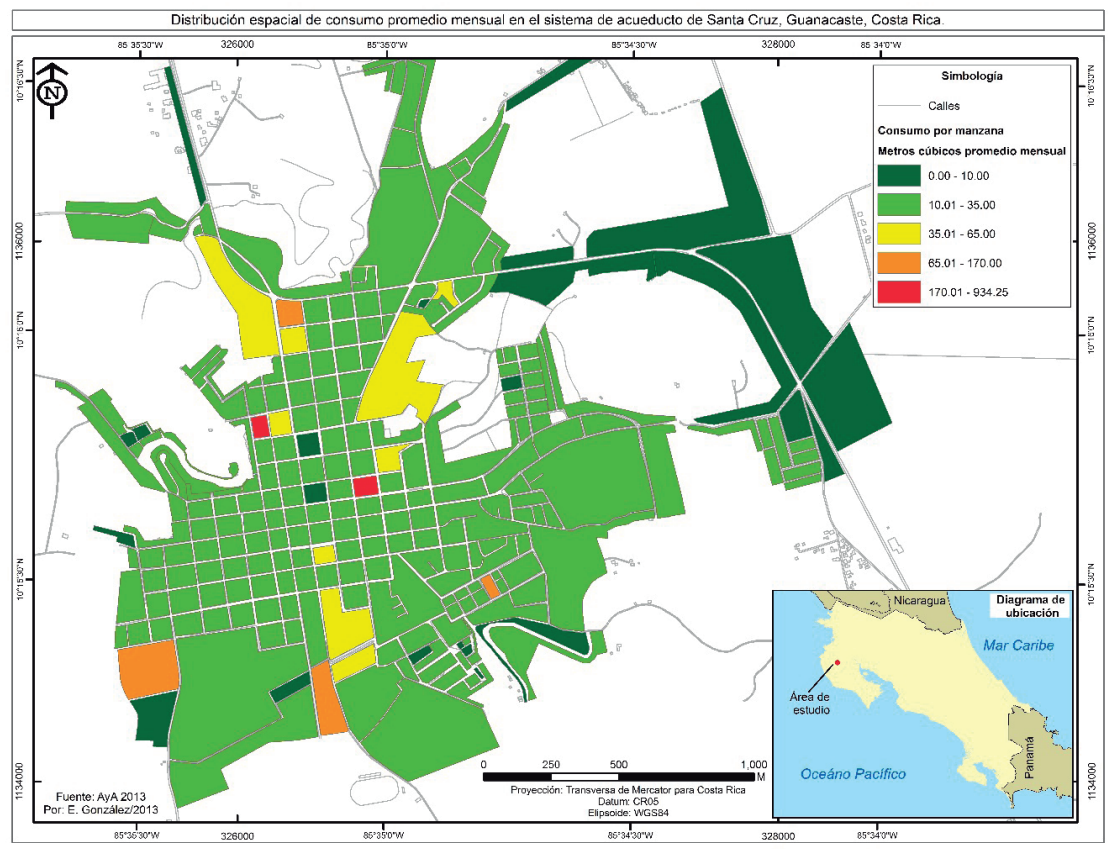

Fuente: AyA 2012. Departamento de Desarrollo Físico, UEN Programación y Control.

Un aspecto desarrollado dentro del análisis fue que, con información de diversas fuentes e información creada, se verifican las áreas con potencial a crecimiento urbano, con base en sus características físico-geográficas, y se determinan zonas inadecuadas como áreas de inundación o sitios con presencia de fallas geológicas, además de pendientes del terreno. Este proceso permitió diferenciar áreas con problemas para el crecimiento urbano, y otras que por el contrario presentan características ideales. Con esto se constituye una zonificación preliminar y mediante la asignación 
de la densidad promedio de las manzanas adyacentes se puede tener una idea del crecimiento que tendría el sistema en los próximos años, a la vez, los niveles de inversión necesarios en infraestructura, caudal estimado por área, entre otros.

\section{Proceso de modelado hidráulico}

El modelado hidráulico de redes de distribución de agua potable permite simular el comportamiento de estas a lo largo del día. En el caso específico que se muestra del sistema de Santa Cruz se utilizó el software WaterCAD/GEMS V8i, que es un software comercial para el análisis, modelado y gestión de redes a presión (sistemas de distribución o de riego), desarrollado por la empresa Bentley Systems Incorporated. WaterGEMS permite la simulación hidráulica de un modelo computacional, representado en este caso por elementos tipo: Línea (tramos de tuberías), Punto (nodos de consumo, tanques, reservorios, hidrantes) e Híbridos (bombas, válvulas de control, regulación, etc.)

En un modelo hidráulico se ingresan propiedades del sistema, demandas de agua y reglas de operación del sistema, etc.; con lo que el software calcula caudales, presiones, gradientes hidráulicos, calidad de agua, etc. Un modelo hidráulico sirve entre otras cosas para dimensionar elementos como bombas, tuberías o tanques, calibrar y detectar fugas, analizar la calidad del agua, analizar costos de energía, realizar simulaciones en períodos de horas o días de la red, etc. La confiabilidad de los resultados que se obtienen mediante un modelo hidráulico depende de dos aspectos:

- $\quad$ La incertidumbre de la información base utilizada para generar el modelo.

- $\quad$ El conocimiento adecuado del software utilizado para la modelación.

En otras palabras, no importa que el usuario tenga un alto nivel de conocimiento del software de modelado, si la información base utilizada es de baja calidad, los resultados obtenidos mediante el modelo serán poco precisos. Cuando se construye un modelo hidráulico se busca utilizar la información más precisa disponible, de modo que los resultados generados por el modelo reflejen con mayor exactitud lo que pasa en la realidad. La construcción de un modelo hidráulico se puede dividir en seis fases, las 
cuales pueden variar el orden de ejecución dependiendo de cada modelador. Las fases se muestran en la Figura 5.

Figura 5. Fases en la construcción de un modelo hidráulico confiable.

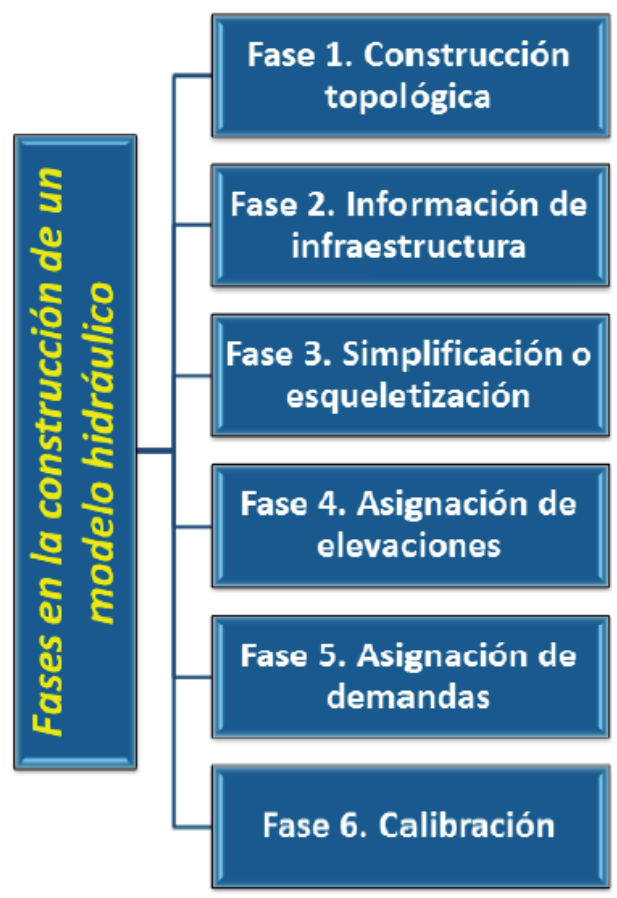

Fuente: Diagnóstico del acueducto de Santa Cruz y propuestas de mejora. Bejarano, 2013.

La primera fase es la construcción topológica de la red, la cual consiste en dibujar a escala todas las tuberías que conforman la red de distribución. El proceso usual que se utilizaba antes era cargar los planos del sistema como imagen de fondo en el software de modelado, y a partir de ahí proceder a dibujar una por una las tuberías que conforman el sistema.

La segunda fase, información de infraestructura, consiste en incluir todos los elementos adicionales a las tuberías como tanques, pozos, válvulas, etc., además de características específicas de operación de dichos elementos, como, por ejemplo, curvas características de las bombas, niveles de operación de tanques, estado de las válvulas (abierta, cerrada o regulada), etc. Es 
también en esta fase donde se suele asignar a cada tubería las características específicas como diámetro, material y coeficiente de rugosidad.

WaterGEMS cuenta actualmente con una herramienta llamada Model Builder, que de forma automática ejecuta la fase 1 y 2 del proceso de modelado de un sistema, utilizando para esto archivos base que pueden tener diferentes formatos. Para construir el modelo del acueducto de Santa Cruz se utilizó la herramienta Model Builder. Básicamente, la herramienta sirve para construir el modelo de forma automática, importando datos desde otros tipos de archivo, entre los más conocidos y utilizados se pueden mencionar archivos tipo CAD, ESRI Shapefiles, archivos dBase, Excel, EPAnet y también archivos de versiones más antiguas de WaterCAD. Se utilizó como base el archivo de AutoCAD con los planos de la red de Santa Cruz, y por medio del Model Builder se generó automáticamente el dibujo de la red a escala.

Model Builder lleva a cabo, automáticamente, tanto el proceso de dibujar la red como la etapa de asignar la información de infraestructura (para los elementos tipo tubería), sin embargo, esta segunda etapa puede incorporar muchos errores en el modelo dependiendo de la calidad y detalle la información base que se utilice. Entre más actualizada y detallada se encuentre la información base, más rápida y simple se puede finalizar la fase de construcción del modelo, además de generar resultados mucho más confiables con el mismo. En la Figura 10 se muestra el modelo que se obtuvo luego de utilizar la herramienta Model Builder para convertir la información CAD en el modelo hidráulico en WaterGEMS.

La tercera fase de construcción de un modelo no incorpora información adicional, por el contrario, busca eliminar parte de la información para simplificar el proceso de modelado. La Esqueletización o Simplificación (Skeletonization) es el proceso de seleccionar las partes de la red que tienen un impacto significativo en el comportamiento del sistema y modelar solamente estos elementos, eliminando todos los demás. En sistemas de gran tamaño puede ser muy complicado añadir al modelo cada uno de los elementos que lo conforman, además, que esto requiere mucho tiempo, por lo que muchas veces se elimina gran parte de la red y se modela solamente líneas principales o las que tienen influencia directa en lo que se quiere modelar.

Crear un modelo de un sistema muy grande, con gran cantidad de detalles y elementos, sin aplicar una Simplificación, podría generar muy 
poca diferencia en los resultados con un modelo que se haya simplificado previamente.

El nivel de Esqueletización de un modelo depende directamente del propósito para el cual se genera el modelo. Por ejemplo, para un análisis de costos de energía es preferible un modelo con alto grado de simplificación, pero un modelo creado para un análisis de flujo para incendio, o un análisis de calidad de agua, es mucho más adecuado contar con mayor nivel de detalle, por lo que es recomendable un bajo grado de esqueletización. En resumen, el grado de esqueletización que puede tener un modelo lo define el usuario, sabiendo previamente con qué objetivo se está construyendo el modelo y los resultados que se quieren obtener.

Figura 6. Modelo hidráulico de Santa Cruz obtenido con Model Builder.

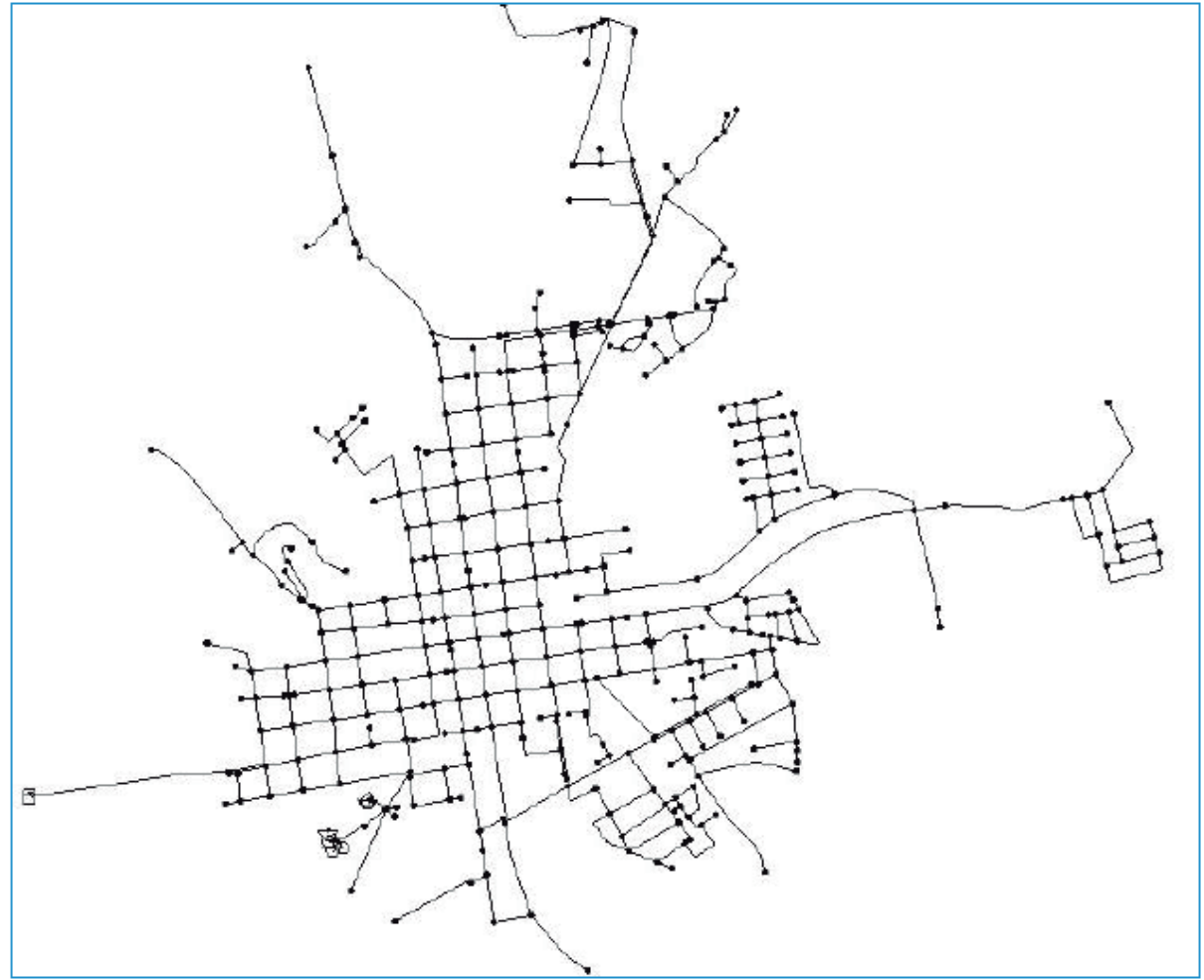

Fuente: Diagnóstico del acueducto de Santa Cruz y propuestas de mejora. Bejarano, 2013. 
La cuarta fase de elaboración de un modelo consiste en la asignación de elevaciones a todos los nodos o elementos tipo nodo del sistema. El valor de elevación en los nodos no es necesario para que el modelo resuelva las diferentes ecuaciones de solución de la red, ya que se resuelven utilizando la línea de gradiente hidráulico (HGL por sus siglas en inglés), sin embargo, una vez que el HGL es calculado, es cuando se necesita el valor de la elevación del nodo para poder obtener el valor de presión en el mismo. En caso de que el usuario sólo necesite calcular caudales, velocidades y gradientes hidráulicos, no será necesario contar con los datos de elevación, sino que se puede trabajar simplemente con diferencias de elevación entre puntos (por ejemplo, un perfil topográfico). Para el caso de Santa Cruz es necesario indicar el valor de elevación, ya que las mediciones de campo utilizadas para la calibración, y en general para observar el comportamiento de la red, miden valores de presión y no HGL.

La calidad de la información base utilizada para obtener las elevaciones tiene un impacto directo en los resultados obtenidos mediante el modelo. Entre mayor sea la incertidumbre de los datos utilizados, mayor será la incertidumbre en las presiones obtenidas luego de una simulación. Para el caso de Santa Cruz, ya fue mostrado en la sección anterior el Modelo de Elevación Digital (MED) que fue creado. En la Figura 7 se muestran las curvas de nivel que se obtienen de dicho MED. Se aprecian pocas curvas, ya que esta zona es sumamente plana, por lo que se vuelve prioritario que la información sea de buena calidad.

Una vez que se cuenta con un archivo de curvas de nivel, o un MED confiable, WaterGEMS cuenta con una herramienta llamada TRex, la cual simplemente toma las elevaciones del archivo base que se le asigne de referencia y las interpola a los nodos y elementos del sistema, incluyendo nodos de consumo, tanques, bombas, etc.

La quinta fase, asignación de la demanda de agua, es una de las fases que se ven más beneficiadas con el uso de Sistemas de Información Geográfica (SIG). En un sistema de abastecimiento de agua potable la demanda de agua no es constante, sino que por el contrario varía a lo largo de todo el día, ya que las actividades de las personas y los hábitos de consumo son variables de acuerdo con las costumbres del lugar. Además, la variación se da espacialmente, es decir, unas zonas del sistema consumen más que otras, por ejemplo, un sector donde haya concentrado muchos 
Figura 7. Curvas de nivel de la zona de Santa Cruz.

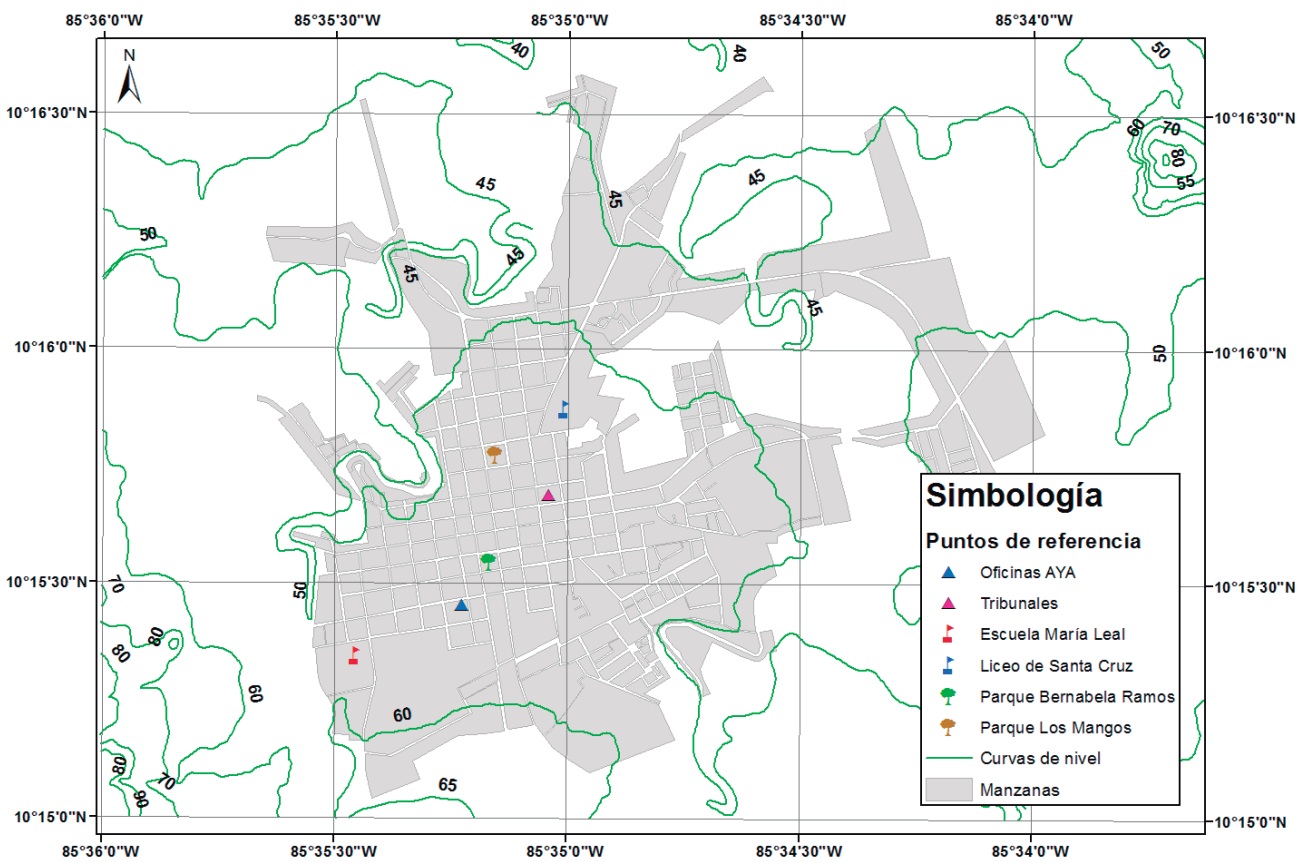

Fuente: Diagnóstico del acueducto de Santa Cruz y propuestas de mejora. Bejarano, 2013.

negocios comerciales tipo restaurantes, hoteles, etc., van a tener mayor consumo a lo largo del día que una zona residencial donde probablemente el consumo sea bien temprano en la mañana y por las tardes o noche. Para obtener resultados más precisos mediante un modelo hidráulico conviene simular estas condiciones de variación de la demanda, para lo que se lleva a cabo lo que se denomina una Simulación en Período Extendido (EPS por sus siglas en inglés).

Una EPS se lleva a cabo de la siguiente manera: se le asigna un caudal promedio a cada nodo del sistema de primero, y posteriormente, se le asigna una curva de variación horaria de la demanda, en la cual básicamente se indican factores de multiplicación a la demanda promedio para incrementar o reducir la demanda en una hora específica. Para obtener la curva horaria del sistema es necesario realizar mediciones de caudal en uno o varios puntos dependiendo del sistema. La curva de variación horaria de Santa Cruz se muestra en la Figura 8. 
Esteban Alberto González-Ramírez - Esteban Bejarano-Salazar

Sistemas de información geográfica y modelado hidráulico de redes de abastecimiento de agua potable: estudios de caso en la provincia de Guanacaste, Costa Rica.

Figura 8. Curva de variación horaria de la demanda en Santa Cruz.

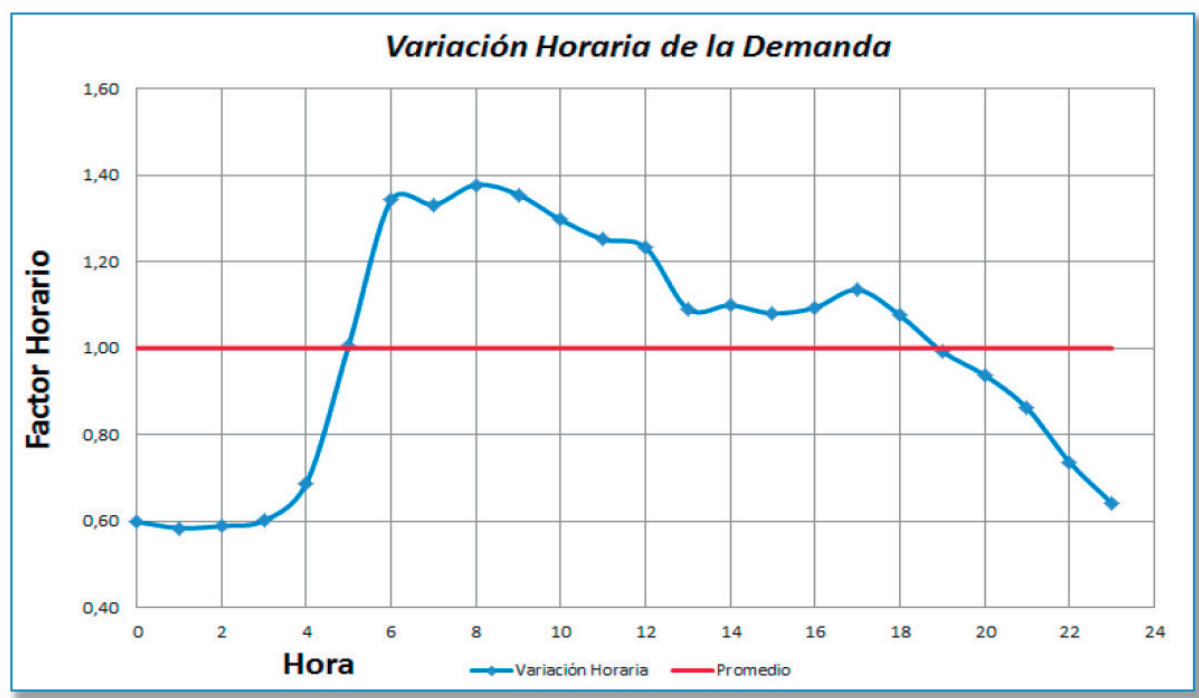

Fuente: Diagnóstico del acueducto de Santa Cruz y propuestas de mejora. Bejarano, 2013.

La Figura 12 muestra que en Santa Cruz la demanda se incrementa considerablemente a partir de las 5:00 am hasta alcanzar el pico a las 8:00 am, y de ahí se empieza a reducir hasta el punto más bajo en horas de la madrugada.

La parte que se torna sumamente compleja es la asignación de la demanda promedio a cada nodo del sistema, sin embargo, si se utilizan SIG, WaterGEMS cuenta con una herramienta llamada Load Builder, la cual simplifica este proceso considerablemente. Load Builder es una herramienta creada con el objetivo de facilitar el proceso de asignar la demanda en los nodos de forma rápida, a partir de bases de datos georreferenciadas. Existen 9 métodos diferentes que se pueden aplicar para cargar la demanda a los nodos. La escogencia de un método u otro depende de la forma en que se tengan organizados los datos de consumo. Estos 9 métodos se pueden agrupar en 3 categorías según la forma en que se importan los datos de consumo a los nodos:

\begin{tabular}{|l|l|c|} 
1. Datos puntuales a nodo & 2. Polígonos de flujo a nodo & $\begin{array}{c}\text { 3. Polígonos de población/ } \\
\text { uso del suelo a nodo. }\end{array}$
\end{tabular}


La categoría de datos puntuales a nodo, como su nombre lo indica, se utiliza cuando los datos de consumo se tienen de forma puntual, por ejemplo, un Shapefile con todos los micromedidores del sistema y su consumo o demanda promedio. Para esta categoría existen 3 métodos de asignación de demanda: Nodo cercano, Tubo cercano o Puntos en polígonos de servicio.

La categoría de polígonos de flujo a nodo se utiliza cuando los datos de consumo se tienen por áreas definidas y no puntualmente, por ejemplo, el consumo promedio de cada manzana de facturación de un pueblo o ciudad. En este caso la asignación se puede llevar a cabo mediante 3 métodos distintos: Distribución promedio, Distribución proporcional al área y Distribución proporcional a la población.

La categoría de polígonos de población o uso del suelo es un poco más complicada que las dos anteriores, pero en general, como su nombre lo indica, se utiliza cuando se cuenta con datos bastante confiables de distribución de la población o uso del suelo.

En Costa Rica no se cuenta aún con bases de datos georreferenciadas de cada micromedidor en un sistema. Lo más usual es dividir los sistemas en sectores de facturación, y asignar un número a cada manzana de facturación dentro de un sector, por lo que, a la hora de hacer la lectura de un micromedidor específico, este se asocia a esa manzana para poder ubicarlo espacialmente. Se debe entender por manzana en este caso un área específica que abarca muchos servicios, que en zona urbana, generalmente coincide con las "manzanas" o "cuadras" como se les conoce comúnmente, sin embargo, en zonas rurales una manzana puede ser un área mucho más extensa que agrupa varios servicios. Por esta razón, es que el método de asignación por polígonos de flujo se considera el más adecuado para la asignación de la demanda en estos casos. Para el caso del acueducto de Santa Cruz se utilizó el método de Distribución Proporcional al Área. Esta decisión, como se explicó, se basa en que el nivel más detallado de información que se puede asociar entre la cartografía y la base de datos comercial de AyA es a nivel de manzanas de facturación. Para poder utilizar el método de Distribución Proporcional al Área se necesita asignar un valor de caudal promedio a cada manzana. Para generar este valor de caudal se llevó a cabo el siguiente procedimiento: 
- De la base de datos comercial de AyA se obtuvo el consumo mensual total por manzana, para cada uno de los meses del año 2011. A partir de esta información se calculó el consumo mensual promedio de cada una de ellas.

- $\quad$ La información del consumo mensual promedio se agregó a un Shapefile debidamente georreferenciado, esto con el fin de ubicar espacialmente cada manzana del sistema.

- $\quad$ Se calculó el porcentaje de consumo relativo, es decir, el porcentaje de consumo respecto al consumo total del sistema. Para esto se divide el consumo promedio de cada manzana entre la sumatoria de los consumos promedio total del sistema.

- $\quad$ Para obtener el caudal promedio de cada manzana se multiplicó el caudal promedio diario del sistema por el porcentaje de consumo relativo obtenido en el punto 3. Este cálculo permite distribuir el caudal promedio diario según los porcentajes de consumo medidos, por lo que las manzanas con mayor consumo medido presentan mayor caudal y las de menor consumo caudales más bajos. En la Figura 9 se muestra la distribución de caudales por manzana:

Lo segundo que se necesita para poder utilizar el método de Distribución Proporcional al Área es definir áreas de servicio para cada nodo. Los nodos del modelo deben tener delimitada un área de servicio para que Load Builder pueda realizar el cálculo del caudal que demanda cada nodo. Para delimitar áreas de servicio en WaterGEMS, la forma más rápida y simple es generar Polígonos de Thiessen, esto debido a que el programa cuenta con una herramienta que los construye de forma automática (Thiessen Polygon). Los polígonos se crean al unir mediante rectas todos los nodos del modelo y luego se traza la mediatriz de las rectas o segmentos de unión. Las intersecciones de las mediatrices determinan una serie de polígonos alrededor de los nodos de manera que el perímetro de los polígonos sea equidistante a los nodos vecinos. Aplicando la herramienta Thiessen Polygon se generan polígonos automáticamente alrededor de todos los nodos del modelo, estos se muestran en la Figura 10. 
Figura 9. Distribución de caudales por manzana

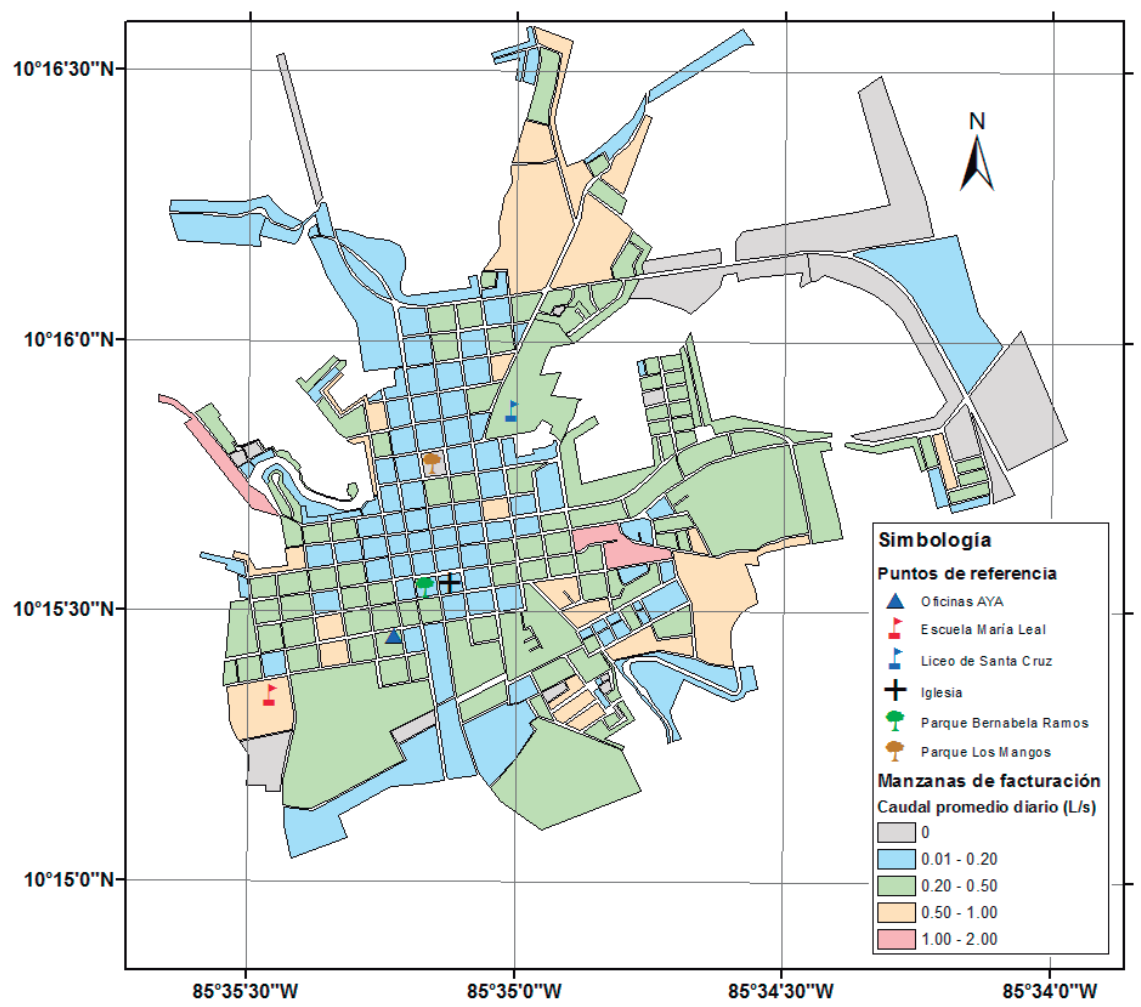

Fuente: Diagnóstico del acueducto de Santa Cruz y propuestas de mejora. Bejarano, 2013.

Una vez listo el archivo de demanda promedio por manzana y definidas las áreas de servicio, Load Builder lleva a cabo la distribución automática de la demanda a los nodos del modelo. En la Figura 11 se muestra el resultado de los caudales asignados a cada nodo del modelo.

La última fase en la construcción de un modelo hidráulico es la calibración de este.. La etapa de calibración es una fase donde se hacen variaciones al modelo creado para obtener resultados deseados en ciertos puntos de control del sistema, sin embargo, ya que ese no es el objetivo de este estudio, no se detalla aquí el procedimiento que se utilizó para calibrar 
Figura 10. Polígonos de Thiessen que delimitan áreas de servicio para cada nodo.

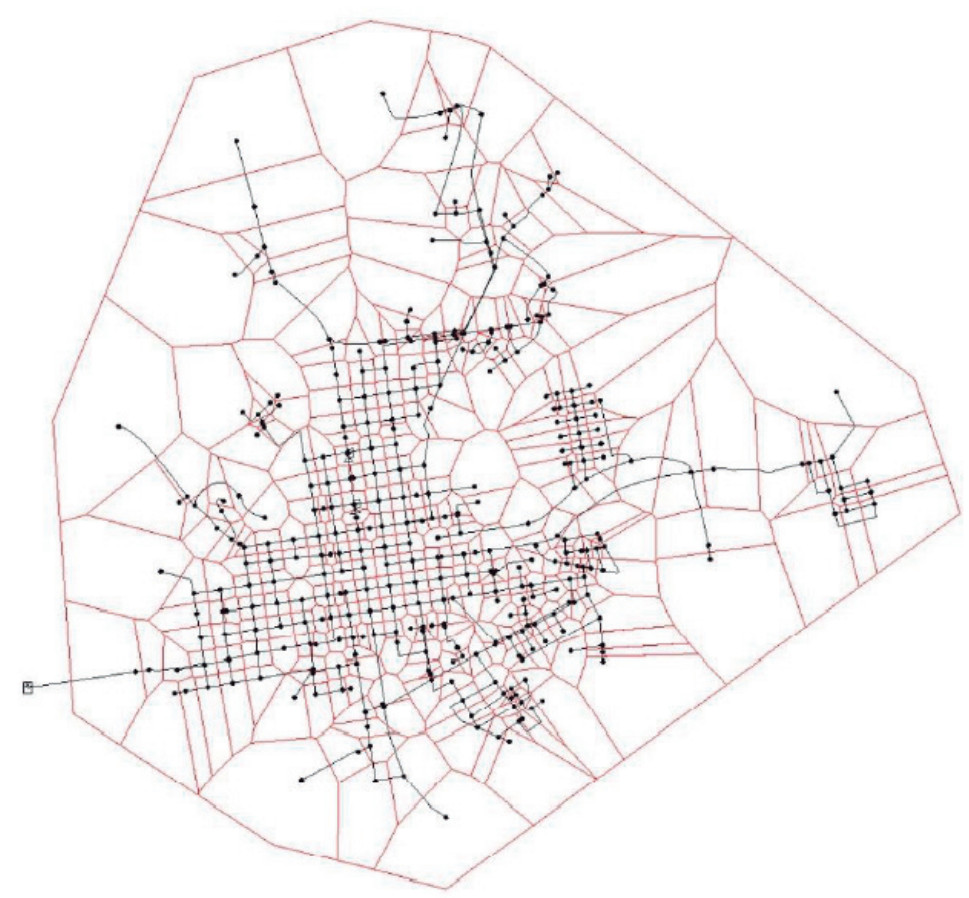

Fuente: Diagnóstico del acueducto de Santa Cruz y propuestas de mejora. Bejarano, 2013.

el modelo de Santa Cruz. Por último, se ha logrado observar en la práctica que los resultados iniciales de un modelo se mejoran sustancialmente cuando se utilizan herramientas como Model Builder, TRex, o Load Builder, las cuales se basan en incorporar al modelo de forma rápida y sencilla la información previamente preparada en otros formatos, como lo son los archivos generados con SIG. Al obtener resultados iniciales mucho más similares a la realidad, el proceso de calibración se puede llevar a cabo de forma más rápida y sencilla, hasta obtener un grado de precisión aceptable. 
Figura 11. Demanda distribuida a los nodos.

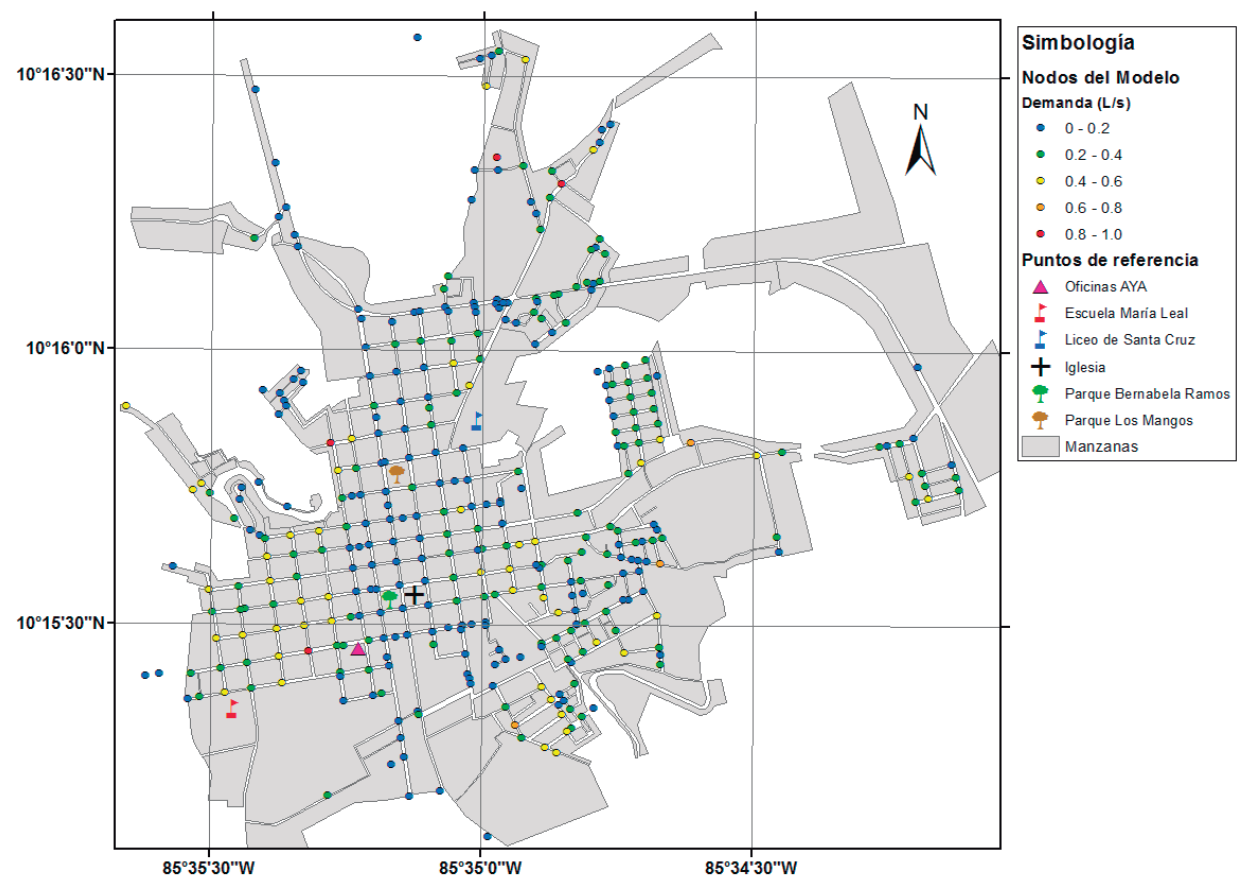

Fuente: Diagnóstico del acueducto de Santa Cruz y propuestas de mejora. Bejarano, 2013.

\section{Resultados y conclusiones}

Con el trabajo realizado, se ha logrado sistematizar una metodología que incluye el tratamiento y gestión de la información de los sistemas de acueducto y saneamiento, de manera georeferenciada y ajustada a los estándares institucionales de este tipo de datos espaciales, y su incorporación para su uso en un software de modelado hidráulico. Este resultado ha conseguido constituir una sincronía entre las utilidades de los SIG y los métodos de modelado hidráulico, ambos utilizados por el AyA, con productos cartográficos y de simulación de alta calidad, y con esto obtener una majora en el servicio que se presta a la población.

El proceso permitió sintetizar en capas organizadas, toda la información disponible pertinente al sistema de Santa Cruz y del nuevo proyecto de abastecimiento de Playa Panamá-Trancas en Papagayo Norte. Los datos incluyen todo un inventario de tuberías, accesorios e infraestructuras con una base de datos estructurada para cada entidad. 
La información generada tiene diversas funcionalidades y se convierte, por ejemplo, en herramienta para la operación y mantenimiento, planeación territorial, gestión municipal, aplicación en aspectos jurídicos, entre otros varios. Esta información se distribuye a niveles superiores del AyA y mediante informes se distribuye y publicita lo realizado.

Para el sistema Santa Cruz, la información generada provee la capacidad de ampliar el ámbito de influencia para su uso en diversos estudios y proyectos, y no solo alguna problemática parcial. Permite también mayor coordinación y capacidad de divulgación, debido a que los formatos utilizados son ampliamente difundidos y su uso se facilita en sobremanera.

Con la generación de esta información la Dirección de Desarrollo Físico puede brindar un aporte importante a una gran variedad de responsabilidades institucionales, además, la información permite contribuir con diversos organismos, como municipalidades, ministerios, instituciones públicas, entre otras, a contar con información cartográfica de calidad sobre la infraestructura instalada, y con esto facilitar la gestión de las diversas áreas a nivel nacional que demandan este tipo de datos.

También, la construcción de la información permite, entre otros, ayudar a superar las trabas creadas ante el manejo de información parcial, fragmentada, inconsistente y que no cuenta con todos los criterios cartográficos modernos (generados por el IGN y las escuelas de Geografía de las universidades nacionales). La estandarización de la información con base en la cartografía oficial permite que no se derrochen energías en la duplicación de tareas, incluso dentro de un mismo departamento o UEN.

En el ámbito de la UEN, lo anteriormente descrito, contribuye notablemente a la construcción de simulaciones hidráulicas más precisas, ya que se cuenta con información base cartográfica de mejor calidad y de mayor apego a la realidad, y una base espacializada de los sistemas de acueducto y saneamiento con referencia geográfica, proyección y corrección geométrica.

El modelado hidráulico tiene un avance importante, ya que la información base que usan los programas computacionales de simulación es más exacta, lo que notablemente trae consigo un mejoramiento en la calidad de los resultados. Los datos provenientes del SIG logran tener en poco tiempo una cantidad de información de los sistemas y el territorio que 
Esteban Alberto González-Ramírez - Esteban Bejarano-Salazar Geographic information systems and hydraulic modeling of potable water supply networks: case studies in the province of Guanacaste, Costa Rica.

ocupan, como topografía del terreno, carreteras, límites físico-geográficos, entre otros, lo que facilita la toma de decisiones.

\section{Referencias}

Bejarano, E. (2013). Diagnóstico del acueducto de Santa Cruz y propuestas de mejora. (Proyecto de graduación para obtener el grado de Licenciatura en Ingeniería Civil, Escuela de Ingeniería Civil), Universidad de Costa Rica, San José, Costa Rica.

Bentley Corp. (2012). Recuperado el 10 de julio de 2012 del sitio web de Bentley Corp.: http://docs.bentley.com/es/InRoads/InRoads_Suite_Help/reference_information/file/new/typesofsurfacefiles.htm

Buzai, G. D. (2011). Geografía y Sistemas de Información Geográfica (Evolución teórico-metodológica hacia campos emergentes): Conferencia Magistral XIII EGAL Costa Rica 2011. Universidad Nacional de Luján, Programa de Estudios Geográficos, Argentina.

Cervera, B. y Rodríguez, J.A. (2008). Captura de información alfanumérica. México: Alfaomega.

Comas, D. y Ruiz, E. (1993). Fundamentos de Sistemas de Información Geográfica. Barcelona: Editorial Universidad de Barcelona, España.

Foresman, T. (1998). The history of the geographic information systems: pespectives from the pioneers. Prentice Hall: Upper Sanddle River. USA.

Instituto Costarricense de Acueductos y Alcantarillados (AyA) (2010b). Datos de inversión, rezago y proyectos de los sistemas AyA. Subgerencia de Ambiente, Investigación y Desarrollo, Pavas, Costa Rica.

Instituto Tecnológico de Costa Rica (2008). Atlas cantonal digital. ITEC. Cartago, Costa Rica.

Moreno, A. (2008). Sistemas y análisis de la información geográfica: manual de autoaprendizaje con ArcGIS. México: Alfaomega Grupo Editor.

O`Sullivan, D. y Unwin, D. (2003). Geographic Information analysis. Hoboken (NJ): John Wiley and Sons. New York, USA.

Rodríguez, J.A. (2008). El modelo de datos vectorial: características y formatos. México: Alfaomega. 